\title{
MODIFIED SCLERAL BUCKLING WITH A NON-CONTACT WIDE ANGLE VIEWING SYSTEM AND A 25G CHANDELIER ENDOILLUMINTOR
}

\author{
Tehmina Jahangir, ${ }^{1}$ Haroon Tayyab, ${ }^{2}$ Muhammad Naeem, ${ }^{3}$ Qasim Lateef, ${ }^{4}$ Asad Aslam Khan ${ }^{5}$
}

\begin{abstract}
Purpose: To evaluate the outcome of scleral buckling surgery using a wide - angle non-contact viewing system and chandelier endoillumination for per-operative fundus visualization in patients with non-complex rhegmatogenous retinal detachments.
\end{abstract}

Materials and Methods: This was a prospective, interventional study carried out at the Department of Ophthalmology, Mayo Hospital Lahore over a period of six months. Non-probability convenience sampling technique was employed. Fifteen eyes of fifteen patients underwent modified scleral buckling procedure for rhegmatogenous retinal detachment using a $25 \mathrm{G}$

\section{Jahangir T. ${ }^{1}$}

Vitreo-Retinal Fellow, KEMU / Mayo Hospital, Lahore

Tayyab H. ${ }^{2}$

Vitreo-Retinal Fellow, KEMU / Mayo Hospital, Lahore

Naeem M. ${ }^{3}$

Vitreo-Retinal Fellow, KEMU / Mayo Hospital, Lahore

Lateef $\mathrm{Q}{ }^{4}$

Assistant Professor Ophthalmology

KEMU / Mayo Hospital, Lahore

Khan A.A. ${ }^{5}$

Head of Ophthalmology Department

KEMU / Mayo Hospital, Lahore
Awh Chandelier (inserted into the sclera through the pars plana) and wide angle viewing system to view the fundus intraoperatively instead of the conventional Indirect Ophthalmoscope.

Results: The mean age of the patients in this study group was $41.9 \pm 12.4$ years. Out of fifteen patients, $13 \mathrm{had}$ flat retinas postoperatively. Two patients had to undergo pars plana vitrectomy with silicone oil tamponade due to development of retinal detachment secondary to PVR.

Conclusion: Modified scleral buckling with the concurrent use of a chandelier light and wide angle viewing system provides an easier and more convenient means of visualizing the fundus under panoramic viewing conditions intraoperatively.

Key Words: Wide - angle viewing system. Retinal detachment. Scleral buckling. Endoillumination.

\section{Introduction}

There are three procedures commonly used for repair of primary rhegmatogenous retinal detachment: conventional buckling, pneumatic retinopexy, pars plana vitrectomy or a combination of these procedures. ${ }^{1,2}$ With advances in surgical techniques and instrumentation, vitrectomy is being used more frequently in the initial management of retinal detachments.

However, scleral buckling is a time - tested and widely prevalent treatment modality used for primary rhegmatogenous retinal detachment. ${ }^{3}$ It is usually car- 
ried out with the aid of a binocular ophthalmoscope and a condensing lens. The recent widespread use of chandelier illumination in conjunction with a wideangle viewing system enables the entire buckling procedure to be performed through the microscope without the burden of repeated wearing and removal of binocular ophthalmoscope. ${ }^{4}$ Additionally, focus and magnification adjustment under the surgical microscope enable per-op identification of previously unrecognized tears. ${ }^{5}$ The quality and angle of view of the fundus through a surgical microscope with chandelier endoillumination is panoramic and erect compared to that observed through the conventional binocular ophthalmoscope via the condensing lens. ${ }^{6,7}$

In this article, we present a series of cases in which patients underwent scleral buckling under the microscope using the Oculus Binocular Indirect Ophthalmo Microscope (BIOM) and a single 25 gauge Awh chandelier for illumination. This idea allows us to perform scleral buckling procedures in relatively fresh retinal detachments using the same viewing systems as used for vitrectomy procedures.

The theoretical concerns of the current procedure may include bacterial inoculation into the vitreous cavity during transconjunctival insertion of the chandelier fiber tip and vitreous incarceration to the sclerotomy after the fiber removal. In my opinion, careful disinfection of the ocular surface by repeated irrigation with diluted povidone - iodine and the use of a stepped incision for the self - retaining cannula is effective against endophthalmitis in this series.

\section{Materials and Methods}

Study Design: A prospective interventional study.

Subjects used: 15 eyes of 15 patients.

Sampling Strategy: Non-probability convenience sampling.

Settings: Department of Ophthalmology, Mayo Hospital Lahore.

Duration of Study: 6 months.

\section{Inclusion Criteria}

- Patients with Rhegmatogenous Retinal Detachment of recent onset having a single peripheral break.

\section{Exclusion Criteria}

- Patients with media opacities such as vitreous hemorrhage or significant cataract.
- Patients with PVR equal to or greater than grade B.

- Patients with coexisting ocular pathology such as glaucoma and uveitis.

- Patients with multiple breaks (separated by more than 90 degrees).

\section{Procedure Followed}

After obtaining ethical approval from the hospital's ethical committee and informed consent from the patients we analyzed 15 eyes of 15 patients undergoing endo-illumination assisted scleral buckling for primary rhegmatogenous retinal detachment fulfilling the above criteria. The study was carried out over a 6month period in a prospective, non-randomized manner at Ophthalmology department, Mayo Hospital Lahore.

Following enrollment, all patients underwent complete preoperative assessment including ocular and systemic disease history, symptoms and measurement of Snellen best - corrected visual acuity. Slit - lamp examination was also performed, including assessment of the anterior segment, and intraocular pressure (IOP) measurement. Fundus examination with slit-lamp biomicroscopy and indirect ophthalmoscopy was performed to evaluate the extent of retinal detachment and the presence of any predisposing pathologic features in the peripheral retina. The fundus / slit - lamp exam was also used to grade PVR, to detect signs of myopic degeneration, to locate retinal breaks, and to determine their location, type, and number.

All patients were operated under peribulbar anesthesia. After a $360^{\circ}$ limbal peritomy, traction sutures were passed under the rectus muscles. The scleral band was passed under the muscles, secured with a Watzke sleeve and secured with 5/0 ethibond in all four quadrants.

We achieved fundal view with the help of an Awh $25-\mathrm{g}$ self - retaining endoilluminator connected to a photon (Synergetics) light source and a wide - angle viewing system, in this case the BInocular OphthalmoMicroscope (BIOM; Oculus, Germany). The 25G Awh chandelier (Figure 1) is designed to be inserted through a transconjunctival $25-\mathrm{G}$ needle insertion. ${ }^{8,9}$ The light was turned off until after the chandelier was inserted. A $25 \mathrm{G}$ stepped needle puncture was created through the pars plana, approximately 120 degrees away from the break site and 3.5 or $4.0 \mathrm{~mm}$ from the limbus in pseudophakic and phakic eyes respectively. The needle was removed from the eye but it is essen- 
tial not to look away from the puncture wound. ${ }^{8}$ Without looking away from the puncture site, the conical tip of the chandelier was inserted through the sclerotomy. Gentle pressure was applied to stabilize the eye with one hand and with the other, the chandelier was pressed firmly towards the center of the eye. The fiberoptic was connected to a photon light source. Initially, the needle was inserted obliquely into the sclera at an angle of about $30^{\circ}$ to $45^{\circ}$, the direction of the needle was then adjusted perpendicular to the sclera as it was inserted into the vitreous cavity. Once the chandelier was inserted, the wide field viewing system was placed on the corneal surface with viscoelastic interface. After visualization was achieved, the image was reinverted using the invertor attached on the microscope. Detailed assessment of the fundus was done using indentation, and the break or breaks were localized. Cryotherapy of the breaks followed by drainage of subretinal fluid (SRF) was performed. The SRF drainage was performed under the operating microscope after swinging the non-contact viewing system out of the observation beam. At this stage, the fundus was checked to confirm retinal flattening along with the desired indentation effect. The band was tightened accordingly to achieve the best possible buckle - break correlation. The chandelier light was removed. After removal using forceps, the site at which the chandelier was placed was closed with a single $6-0$ Vicryl stitch if there was any vitreous herniation.

A drop of povidone - iodine was then instilled followed by conjunctival closure with $6-0$ Vicryl and subconjunctival antibiotic injection.

Patients were examined postoperatively on days 1 , 7, 30, and 180. During each visit, a detailed ophthalmic examination was carried out. Anatomic and functional status of the retina were assessed and IOPs were checked on all visits.

The beauty of this method is that the surgeon is able to perform not only scleral indentation but also exactly localize the retinal break. This procedure thus merges the benefits of greater visualization provided by the endoillumination and wide-angle viewing systems with the security and simplicity of orthodox scleral buckling.

\section{Results}

All patients were followed up for a duration of 6 months. The mean age of the patients in this study group was $41.9+12.4$ years.
Additional retinal breaks within 2 clock hours of the breaks identified preoperatively were identified preoperatively.

The retinal detachment involved the macula in 10 $(67 \%)$ of the eyes.

Out of fifteen patients, $13(87 \%)$ had flat retinas postoperatively (Figure 2). Two patients had to undergo pars plana vitrectomy with silicone oil tamponade due to development of retinal detachment secondary to PVR at the 1 month follow-up visit.

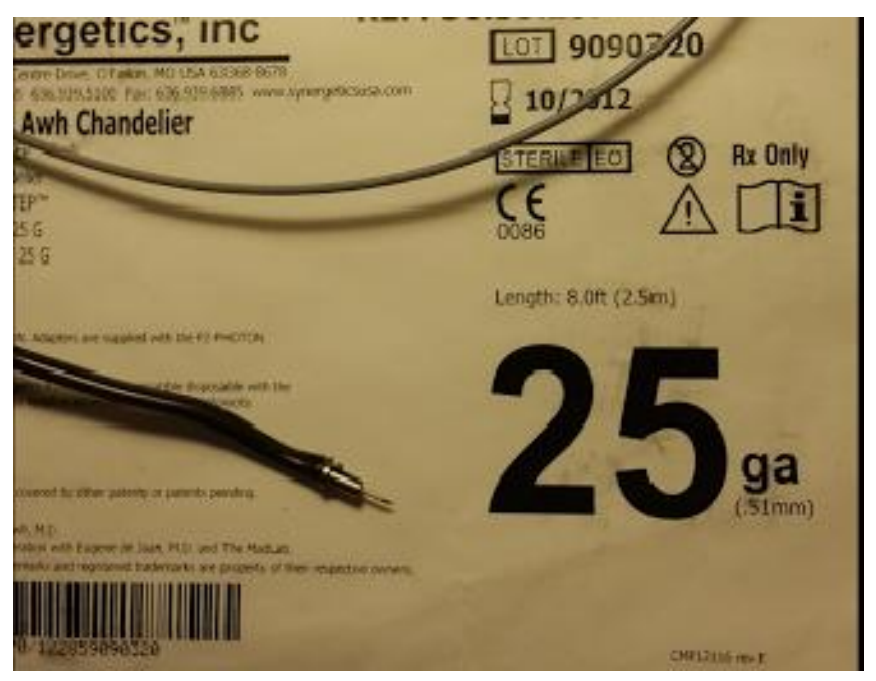

Fig. 1: The 25 gauge Awh Chandelier.

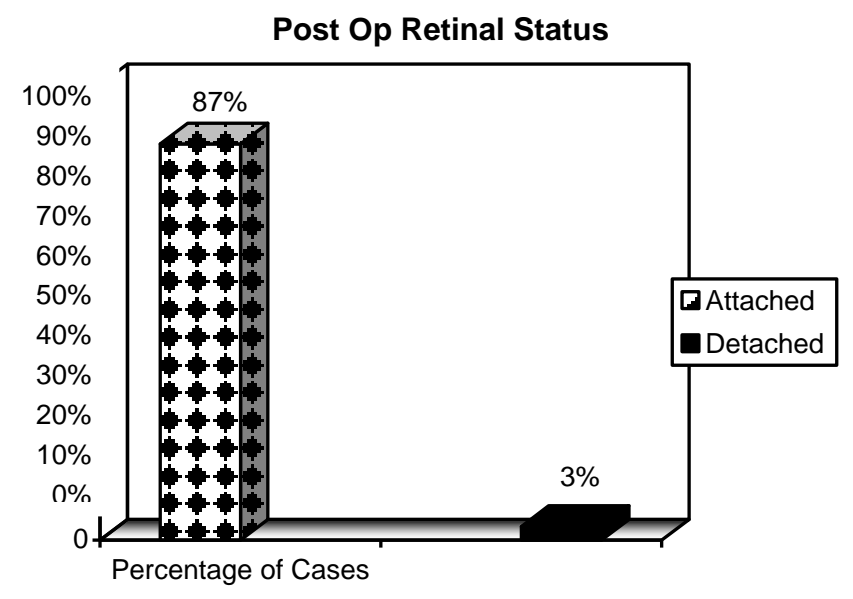

Fig. 2: Post-operative Retinal Status.

The location of the retinal breaks pre-operatively was as follows: superotemporal in 8 (54\%), superonasal in 2 (13\%), inferotemporal in $3(20 \%)$ and inferonasal in $2(13 \%)$ of patients (Figure 3 ). 


\section{Pre-op Location of Retinal Breaks}

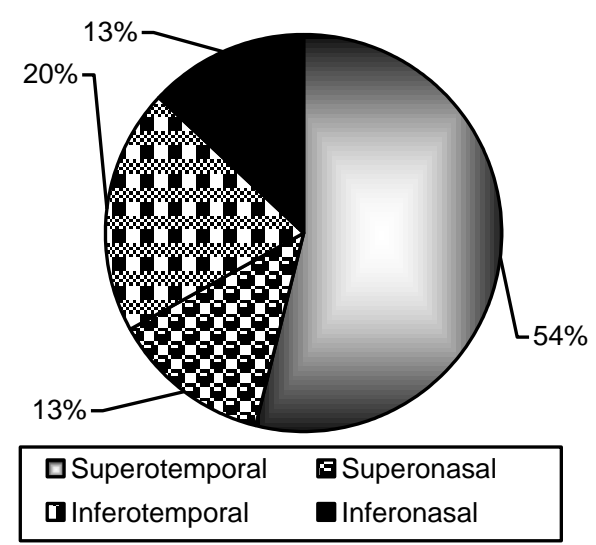

Fig. 3: Pre-operative location of retinal breaks.

There was minor sub-retinal haemorrhage during drainage of SRF in one eye. However, it did not involve the macula, was self - limited and resolved during the follow-up period.

The chi-square statistical test was performed and the p-value was less than 0.05 .

\section{Discussion}

The underlying principle in the procedure of scleral buckling is approximation of neurosensory retina with the retinal pigment epithelium (RPE) by compression of the globe wall thus preventing passage of liquid vitreous into the subretinal space. , $^{3,4}$

Traditional scleral buckling procedure requires repeated use of indirect ophthalmoscope for per-operative fundus visualization. This provides an inverted image which makes performance of simultaneous procedures difficult and requires considerable dexterity. Also repeated donning and removal of the Indirect Ophthalmoscope is not only inconvenient but is also a waste of time. $^{4,7}$

Recent studies have proposed various methodologies for overcoming these issues. Ohji and Tano ${ }^{10}$ have reported the use of a microscope mounted with a wide - field contact lens and a slit-lamp. In addition, Nawrocki et al, ${ }^{11}$ have performed scleral buckling using an Optic Fiber Free Intravitreal Surgical System (OFISS; Topcon, Inc, Paramus, NJ). Aras et al, ${ }^{2}$ have reported a scleral buckling procedure using a torpedo light (Alcon Laboratories, Fort Worth, TX, USA) and a non-contact wide-angle viewing system. The torpedo light is basically a 25 - gauge fiber optic endoilluminator.
The results of our pilot study provide the rationale for evaluating this modified technique for scleral buckling in a larger series of patients to practically and more clearly explain our procedure, which is similar to a normal scleral buckling procedure with the exception that a chandelier is inserted and a non-contact viewing system is used instead of an indirect ophthalmoscope. In terms of post-operative retinal flattening, our results $(87 \%)$ are comparable to those of Aras et al $(81 \%)^{2}$ and Nawrocki et al $(87 \%) .{ }^{11}$ One of our patients had minor sub-retinal bleed at the time of SRF drainage which was comparable to Aras et al. ${ }^{2}$

The use of chandelier light along with a wide-angled viewing system coupled with an image inverter provides an unparalleled erect, panoramic view of the fundus during buckling procedure. ${ }^{12-14}$ The ability to transmit the surgery to an operating room monitor to see every step in fine detail is a significant improvement over operating with an indirect ophthalmoscope, and it may help train surgeons in their process of decision - making during a procedure (i.e., localization, cryotherapy, external drainage).

There was a certain amount of difficulty in moving / rotating the globe to extremes of gaze (in cases of very peripheral breaks) with the chandelier light in place. Also there is the potential for lens touch by the endoilluminator which, however, was not encountered in any of the patients in this case - series.

The chandelier provides a wider field than does a surgical microscope equipped with a slit - lamp, as used by Ohji and Tano. ${ }^{10}$ The chandelier light does not require special equipment such as a slit - lamp or OFISS other than a surgical microscope and a light source. In comparison to the torpedo light used by Aras et al, the chandelier light certain advantages: as the chandelier light is inserted after an angle incision it significantly reduces the risk of vitreous plugging the sclerotomy; also Torpedo light has greater potential for lens damage/touch as compared to a chandelier light. However, none of the cases in our series had lens touch / damage.

We believe that our technique should be easily adoptable by most vitreoretinal surgeons who are familiar with using widefield visualization and that it may help to propagate the art of scleral buckling, which provides excellent results in carefully selected cases. ${ }^{15-}$ 17 However, larger-scale, multi-center, randomized studies are required to establish the safety, efficacy and feasibility of this particular technique.

Thus endoillumination-assisted scleral buckling is 
a novel method enabling conventional buckling to be done using the surgical microscope.

\section{Conclusion}

Modified scleral buckling with the concurrent use of a chandelier light and wide angle viewing system provides a safe and effective means of visualizing the fundus under panoramic viewing conditions intraoperatively.

\section{References}

1. Venkatesh P, Garg S. Endoillumination - assisted scleral buckling: a new approach to retinal detachment repair. Retinal Physician. 2012; 9: 34-37.

2. Aras C, Ucar D, Koytak A, Yetik H. Scleral buckling with a non-contact wide-angle viewing system. Ophthalmologica. 2012; 227: 107-110.

3. C. P. Wilkinson, "Scleral buckling techniques: a simplified approach," in Retina -Vitreous - Macula, D. R. Guyer, L. A. Yanuzzi, S Chang et al., Eds., pp. 12481271, WB Saunders, Philadelphia, Pa, USA, 1999.

4. G. A. Williams and T. M. Aaberg, "Techniques of scleral buckling," in Retina, S.T. Ryan, Ed., pp. 20562065, Elsevier Mosby, New York, NY, USA, $5^{\text {th }}$ edition, 2013.

5. Nam KY, Kim WJ, Jo YJ, Kim JY. Scleral buckling technique using a 25-gauge chandelier endoilluminator. Retina. 2013; 33: 880-2.

6. Tayyab H, Haider MA, Jahangir S, Qureshi BZ. Modified Scleral Buckling Technique Using Endoillumination and Non Contact Wide Angle Viewing System. Pak J Ophthalmol. 2014; Vol. 30, No. 2: 103-107.

7. OluleyeTs, Ibrahim O, Olusanya B. Scleral buckling for retinal detachment in Ibadan, Sub-Saharan Africa: anatomical and visual outcome. ClinOphthalmol. 2013; 7: 1049-52.

8. Awh C. Beginning to see the light. Retinal Physician. November 2010; 28-32.

9. Shiba H, Shiba T, Kodaka R, Shiba K. Retinal reattachment surgery using wide - angle viewing systems and chandelier endoillumination. Folica Japonica de Ophthalmologica Clinica. 2010; 23 (2): 301-304.

10. Ohji M, Tano Y. Vitreoretinal surgery with slit-lamp illumination combined with a wide - angle - viewing contact lens. Am J Ophthalmol. 2004; 137: 955-956.

11. Nawrocki J, Michalewska Z, Michalewski J. Optic Fibre Free Intravitreal Surgical System (OFFISS) in retinal detachment surgery. Ophthalmic Surg Lasers Imaging, 2008; 39: 466-70.

12. Falkner-Radler CI, Myung JS, Moussa S, Paul Chan $\mathrm{RV}$, Smretschnig E, Binder S: Trends in primary retinal detachment surgery: results of a bicenter study. Retina 2011; 31: 928-936.

13. Oshima Y. Chandelier endoillumination in Vitreoretinal Surgery. Retina Today, 2013: 68-72.

14. Sakaguchi H, Oshima Y. Considering the illumination considering the illumination choices in vitreoretinal surgery: continual improvements allow for better, safer outcomes. Retinal Physician, 2012; 3: 20-25.

15. Kita M, Fujii Y, Kawagoe N, Hama S. Scleral buckling with a noncontact wide - angle viewing system in the management of retinal detachment with undetected retinal break: a case report. Clinical Ophthalmology, 2013; 7: 587-589.

16. Nagpal M, Bhardwaj S, Mehrotra N. Throwing new light on buckling surgery. Retina Today. January / February, 2013: 48-52.

17. Oshima Y, Awh CC, Tano Y. Self - retaining 27 gauge transconjunctival chandelier endoillumination for panoramic viewing during vitreous surgery. Am J Ophthalmol. 2007; 143: 166-167. 